\title{
Thermal Characterization of Graphene Based Composites
}

*11 Rathin Sajit, ${ }^{1}$ B.Harinesh, ${ }^{1}$ M.P.Jenarthanan, ${ }^{2} \mathrm{M}$. Ramachandran, ${ }^{2}$ Vidhya Prasanth

${ }^{1}$ SASTRA Deemed University Thanjavur, India

${ }^{2}$ REST Labs, Kaveripattinam, Krishnagiri, Tamil Nadu, India.

*Corresponding author Email: rathinsajit@gmail.com

\begin{abstract}
Graphene, an atomic thin two-dimensional carbonaceous nanomaterial, has exceptional electrical, mechanical and chemical properties. There is also great research interest in the development of two technologies. Since the discovery of graphene, this reliable Wide range of material applications Integrated,and many attempts have been made To modify the structure of graphene. Particular attention is paid. Graphene Derivatives Graphene Oxide Hole Graphene / Graphene oxide, recent Developments development of reduced Graphene oxide and graphene quantum points. In this chapter, the inherent properties of the definition and the different approaches to top-down and basically graphene derivatives are discussed below. This includes the formation of derivatives of graphene by chemical oxidation. In addition, the bit and peel-out mechanism for creating graphene derivatives, which leads For a better understanding of Physics of graphene derivatives And chemical properties.
\end{abstract}

Keywords: Graphene Based Materials, Graphene Based Composites, Thermal Conductivity

\section{Introduction}

Grophene and graphene oxides. However, most of these applications rely on the production of the highest quality synthetic graphene on an industrial scale. Some more advanced applications in nanoelectronics require complex nanoscale structures such as graphene nanoribons. Graphene is two-dimensional sequence Called carbon atoms form in the honeycomb. One square meter of graphene sheet weighs just 0.0077 grams, but can withstand up to four kilograms. I.e. thin and light but incredibly strong. It has a larger surface area, more heat and Has electrical conductivity and various additional incredible properties.That is why scientists and researchers call it "a miracle object". And predicted that it would revolutionize all industries known to man. As mentioned earlier, graphene has many unprecedented properties that can be used in many unusual compounds. The presence of graphene improves the conductivity and strength of the aggregate and helps to form compounds with superior properties. Heat and pressure-resistant and conductive compounds can be formed by graphene in metals, polymers and ceramics.

\section{Graphene Based Materials}

Graphene is the hexagon of atom-thick carbon atoms Is layered. This is the construction module of graphite (used in pencil notes, among others), but graphene is only one notable material - with many astonishing properties, restoring the title. "Miracle Meaning". Repeat for more conductive shaped atoms. [1].The return hexagon is set in Laddu. Graphene is the thinnest material known to humans, the thickness of which is an atomic incredible strength - steel incredibly strong - steel on top of it, Cropon has excellent conductivity and interesting light for heat and electricity. Absorption skills. It is a material that can truly change the world, and has unlimited possibilities to integrate into any industry [2]. Flexible flexible fatigue life improvement that can be achieved with conventional microfiber reinforced polymer compounds using graphene platelets. Ability to spray relatively light weight fractions of graphene compounds $(.20 .2 \%$ by weight of epoxy resin and $.00 .02 \%$ by full laminate fillers) and directly [3]. The quantity designed to directly analyze the surface properties of graphene and eliminate the SBR matrix by minimizing the confusing influence of graphene samples to reduce GO water dispersion is integrated using the reduction conditions previously described [4]. The thus reduced samples were combined with 4 designed quantities of 10: 1 hydrogen hydrate at $90 \mathrm{C}$ to $1: 1$ hydrate at $801 \mathrm{C}$ for $1 \mathrm{~h}$. The thus reduced samples had a 1: 1 hydrate ratio of $80 \mathrm{C}$ at $1 \mathrm{C}$ hydrogen hydrate at $90^{\circ} \mathrm{C} 4$. CoCO3 GNS Growing Solution Introduced Heating System. [5]. Manufactured GNS consists mainly of several Layers that can be determined from AFM images the thickness of the GNS is approximately $0.660 \mathrm{~nm}$, which is related to the 3-layer graphene [6]. Construction teasing due to high electrical conductivity, ultralight weight and thermal stability, home heating and heat injury treatment. Graphene is available in pure form due to its low yield, which is not used in manufacturing (pristine graphene). Furthermore, graphene derivatives such as GO, RGO and FRGO are commonly found and exhibit similar properties to graphene [7]. However, due to heterotomic abnormalities and impurity and structural defects, the reference for materials such as grapheme is theoretically zero dimensional fullerenes. Founded in 1940 as a construction division of Grope Graphite. Zero-dimensional fullerene was established in 1940 as the building block of graphene graphite [8].

The GNFs used in this experiment were chemically synthesized from graphene oxides produced by the Hummer method. The representative image structure of the GNFs. As illustrated in GNFs are a large specific area and two dimensional have high-ratio sheet geometry [9]. The transmission electron microscope of GNFs shows a highly compressed structure. Construction of monographs of large aromatic molecules such as pyrene and PTI Considering the fact that they function as 
blocks. More heat annealing to form graphene films at high temperatures the bottom-up approach can be implemented [10]. Graphite oxide (GO), formerly known as graphite oxide or graphite acid, Made up of different ratios of carbon, oxygen and hydrogen. It is obtained by dissolving graphite in strong metals containing Strong antioxidants and acids [11]. The maximum oxidized total output is between 2.1 and 2.9 at $\mathrm{C}$ : O Is a yellow solid with a ratio It is a layer of graphite Retains, but the aggregate with large and irregular gaps Dissolves spontaneously in the base solution. May scatter or scatter by sound. Pole. Single layer form of graphite Called graphene oxide, which is compared to graphene solvents that form monomolecular sheets. [12] BGO is one of the most To increase the hydrophilicity of the membrane Suitable nanoparticles. The Hummer method in general [46]. GO consists of functional groups, which are further dispersed in polymeric Solution. For water purification technologies, Dip and spin coatings, layer by layer assembly technique Solution [13]. For water purification technologies, the integration of GO in membranes has been enhanced by Dip and spin coatings, layer by layer various methods such as assembly technique and vacuum filtration. Attributes can be achieved. BGO is one of the most Hydrophilicity of the membrane Suitable nana particles to enhance. GO is the norm in the Hummer system Manufactures [14] and has GO functional groups are further dispersed in the polymeric solution. Dip and spin coatings, layer by layer improved properties can be achieved by incorporating GO in membranes through various methods such as assembly. Technique and vacuum filtration. [15]. Pipe systems add additives such as sodium borohydride and ethylene glycol Use reducers, which are either toxic or hygroscopic. Besides, other compilation methods of Sn - GS are difficult Or take time. 4c, d Therefore, it is easier to form Sn-GS to reduce graphene oxide (GO) and It is important to create an environmentally friendly system. According to. [16] .Ruffin's descendants are similar Graphene oxide, graphene and graphene fluoride in various applications can be used as interesting functional properties. However, in this review, only graphene oxide is considered. Extensive discussion of graphene Derivatives can be found in the following publications. [17].

In recent decades, the science and technology of graphene has attracted much attention in the field. To understand the history of some terms of graphene graphite Oxide (GO), graphite peeling, i.e. grapheme oxide, graphite intercalation compounds and grapheme should be discussed first [18]. Initially GO was composed of HNO3 and H2SO4 Integrated by peeling of graphite using. Mechanical behavior of grapheme and graphene oxide. (GO) was intense the following sections were examined using the nanoscale atomic model. However, graphene is one of the (macroscopic) graphene-based compounds The mechanism for making full use of (nana-sized) mechanical properties has not yet been established [19]. the compounds were made using a mixture of commercial TiO2 (P25, Degussa), graphene oxide and one-step gruel with water deionized. Integrated in the laboratory with graphene oxide, and the process is described elsewhere. In a typical preparation, the aqueous dispersion of graphene oxide $(8.4 \mathrm{mg} / \mathrm{ml})$ was dissolved in $200 \mathrm{ml}$ of deionized water. [20]. TiO2 powder (P25, Degussa) was dissolved in deionized water and then added to a solution of graphene oxide. All chemicals used in this study were purchased from Aldrich and Merck chemicals. Made of graphite oxide modified Hummers method. Obtained, after removing the by-products of the reaction Graphite oxide is exposed to PCI analysis. [21]. Ultrasonic bath for at least 2 hours to produce graphene oxide (GO). Finally, the GO suspension was reduced by using hydrazine to obtain reduced graphene oxide (RGO). In some in studies, the GO suspension was centered at different rpm and only the residue obtained at very high rpm (approximately 16000) was used to form RGO layers with some layers of graphene sheets (Rejected due to residue at low rpm) multiple layers of graphene). $0.01 \%, 0.03 \%$ and $0.06 \%$ were added based on weight For GO cement mix in three different percentages. The exact fraction of graphene oxide is as shown in Table 2 Calculated based on the concentration of graphene oxide in the aqueous solution. Stable water in all mixtures [22].

\section{Graphene Based Composites}

A compound (also called a compound or abbreviated compound, a compound (composite) or) Abbreviated compound (also called compound material), is also called. Compound material or abbreviated composition, Common name) made up of two or more. Ingredients. Component materials.[23]. The elements of this element have significantly different chemical or physical properties, and they form a substance with unique properties for individual elements. Within the completion structure, the individual elements are individually and individually, compounds and distinguish compounds from solid solutions [24]. A compound is made up of two or more different substances, stronger than those individual substances. Keep it simple, compounds a Composition of components. Compounds are usually formulated with a specific application in mind such as extra strength, performance or durability. [25]. Aqueous solution was added to the suspension of 2Fe ( $\mathrm{SO} 4) 26 \mathrm{H} 2 \mathrm{O}$ and $\mathrm{NH} 4 \mathrm{H} 2 \mathrm{PO} 4(\mathrm{NH} 4)$ graphene. Nitrogen gas was then added to the aqueous solution mixture of Lion by injection within the solution. Green deposit obtained was washed. Ionized water and separated by a high-speed centrifugal separator.[33]. Microscopic structures of graphene sheets and compounds [26]. The scanning electron microscope (energy spectrum of SEM, FEI, Netherlands, EDS) operates at $20 \mathrm{kV}$, High-Definition Transmission Electron Microscope (powered) HRTEM, JEM2010FEF, JEOLFEF, Japan, Raman spectroscopy (Lab RAMHR,HORIBA Join Y von, France) [27].

Raman laser power $15 \mathrm{~mW}$ and laser excitation 488. The scan was taken in the extended range (1000-3000 cm -1$)$ and the exposure time was $5 \mathrm{~V}$. On the other hand, epoxy compounds containing poorly dispersed RGO were prepared by sonication Ethanol at $60 \mathrm{C}$ for 2 hours [28]. At the same time the ball mill process was not used during the scattering process. In ball mill operations, high cutting pressure is used in the scattering process, when compounds with highly dispersed RGO sheets are expected to be obtained. The mixture was stirred for 12 hours. Graphene is placed in the environment without the PFG properties of grapheme fillers the hierarchical structures of GO / PANI and RGO / PANI compounds described above are mainly used during polymerization [29]. The primarily aniline monomers in graphene sheets form nuclei through p-p contact, responsible for PANI precipitation. We used the powder processing method to create homogeneous and well-dispersed nana 
compounds. Using enrichment with an already prepared graphene suspension DMF solvent Diluted to $1 \mathrm{mg} / \mathrm{ml}$, then rinse for 2 hours with an ultra-sonic bath. [30]. Commercially Available After Sonication - Al2O3 Powder (by AKP-53 Chemicals) Made Sumitomo, particle size $-200 \mathrm{~nm}$, specific area $-12.3 \mathrm{~m} 2 / \mathrm{g}$ ) was added to the suspension. In particular, it exhibits conductive behavior and abnormal area quantum Hall effect, which is why it is used in many technological fields such as Nanocomposites, sensors, batteries and super-capacitors. [31]. High Excellent electrical conductivity due to its remarkable properties including carrier motion $(200,000 \mathrm{~cm} 2 /(\mathrm{v}))$ and better thermal properties. Compared with covalent cross-linking methods, Non-Covalent methods such as hydrogen bonding, contact and $\pi$-a-stacking 3DGPCs can be developed. Are used because the natural structure of graphene is not affected [32]. Also, sometimes non-covalent methods are available. Reversible, it would be highly desirable to retain the inherent properties of creating smart compounds based on graphene or graphene. Two-roll mill EPDM / GnP / MWCNTs compounds were used to create $1 \mathrm{~mm}$ to $1.3 \mathrm{NP}$ clearance at room temperature of $1 \mathrm{~mm}$. [33].

The solid EPDM resin is inserted into the weight plant and formed into a large thin sheet. The first desired amount of Gaps was gradually added to the matrix. X-ray diffraction (XRT) graphite interlocking compound, GNP and M-GNPs and their diffraction technology in nanocomposites using mini material analyzer. Nanocomposites are heterogeneous substances - hence theirs the properties are determined by the same factors as in conventional compounds, viz. Component Properties, Composition, Structure and Interface Links [34]. On the other hand, there the structure is generally more complex than that of micro composites, and it is a polymer /. This is especially true for layered silicate nanocomposites. [26]. Nanocomposites is substances that bind anamorphic particles in the matrix of a fixed substance. The combination of nanoparticles results in a sharp improvement in properties including mechanical strength, hardness and electrical or thermal conductivity. Altman, $\mathrm{n}$. Bukhara, S.M. Wabaidur, s. [35]. At the same time electrochemical detection of ascorbic acid, dopamine and uric acid using Hyder, graphene / quantitative-selective Pt nanocomposites, sensors and accelerator. MoS2 nanocomposites were specifically integrated Summary: $2.2 \mathrm{~g}$ Na2MoO4 \$2 H2O and $2.0 \mathrm{~g} \mathrm{H} 2 \mathrm{NCSNH} 2$ were stirred vigorously in $70 \mathrm{ml}$ of water for approximately $10 \mathrm{~min}$. After adjusting the $\mathrm{pH}$ to less than 1 with $12 \mathrm{M} \mathrm{HCl}$,the mixture was added to $100 \mathrm{~mL}$, converted to defrosted stainless steel autoclave and heated at 200 for $24 \mathrm{~h}$. After cooling naturally, the black MoS2 compounds were collected by filtration[36]. Aqueous pulp processing methods for obtaining uniform and homogeneous dispersions of GPL and Si3N4 ceramic particles prior to compression using SPS. After focusing on $501650 \mathrm{C}$, we detected direct sources of graphene in nanocomposites using Raman spectroscopy. Melting Temperature and torque were continuously recorded during mixing. After preparation, the nanocomposites are further hydrated were placed in the desiccators before further testing to prevent absorption [37].

\section{Thermal Conductivity}

Thermal conductivity is capacity to conduct / transfer heat to a given object. It is usually denoted by ' $\mathrm{k}$ ', but ' $\lambda$ ' and denoted by ' $\kappa$ ', which contact level is called the thermal resistance. Most products are Thermal conductivity Thermal Used in sinks low value Materials as heat conductors are used. [38]Fortier's heat conductivity law (also known as thermal conductivity law) ratio heat transfer. Replaced by an object. This rule is different and the form can be expressed by the following equation. [39]. In the past graphite, diamond and carbon nanotubes have been improved in alloys for decades, and various improved thermal conductivity patterns of polymers and metals have been explored as potential fillers to improve carbon thermal conductivity [40]. Recently, the increase in thermal conductivity and thermal stability of polymers GNPs are some of the graphene made from acid treatment for suspension, which then provides better thermal Heat-peeled layers, epoxy Improvements when embedded in the matrix [41]. Gangly and colleagues dispersed graphene oxide (GO), reacted with Ceylon as an Can be expressed by the following equation. reached $5.8 \mathrm{~W} / \mathrm{mK}$. And $20 \mathrm{wt} \% \mathrm{GO}$. Comparable Under loading conditions, GO polymeric is more likely to form nanocomposites with higher thermal conductivity [42]. Non-oxidized GFs are the most effective offer fillers. Thermal conductivity in low-end pyrolytic GN - GN nanocomposites found in graphite materials is close to thermal conductivity, although the former (unlike the latter) is more mechanical flexible [43]. Therefore, a wide range of GN-GN nanocomposites and significant applications can be considered in heat management and others. Systems [44]. The specific heat is transmitted through the composite frame, which is said to be derived from the calculated theoretical value. To prevent water infiltration due to density Principle of Archimedes compounds using the thinnest Vaseline scale in each sample. [45] Apply a very thin Vaseline film on each sample to prevent density, heat dissipation and water penetration of specific Archimedes after the thermal conductivity of the compounds. The product density, heat dissipation and specificity are then calculated for the thermal conductivity of the compounds [46].

In metals, thermal conductivity caused by free carriers of electrons. Copper is a good heat conductor at room temperature Thermal conductivity $400 \mathrm{~W} \mathrm{~m} \mathrm{-} 1 \mathrm{k}-1$. The density of the product after the thermal conductivity of the compounds [47]. Calculates heat dissipation and output. The thermal conductivity equation (T) lðTP of the sample sheet is determined by аðТЬСгðТЬrðТр, where a (T), Сrð T and $\rho(\mathrm{T})$ are the heat dissipation, respectively. [48]. Indicates the Specific heat capacity and density. Principle of Archimedes compounds using the thinnest Vaseline scale in each sample. Apply a very thin Vaseline film on each sample to prevent density, heat dissipation and water penetration of specific Archimedes after the thermal conductivity of the compounds. The product density, heat dissipation and specificity are then calculated for the thermal conductivity of the compounds [49]. Heat. In metals, thermal conductivity is caused by free carriers of electrons. Copper is a good heat conductor at room temperature Thermal conductivity $400 \mathrm{~W} \mathrm{~m} \mathrm{-} 1 \mathrm{~K}-1$ [50]. Calculates the density, heat dissipation and output of the product after the thermal conductivity of the compounds. The thermal conductivity equation (T) 1ðТр of the sample sheet is determined by аðТЬСrðТЬrðТЬ, where a (T), Crð T and $\rho(\mathrm{T})$ are the heat dissipation, respectively. Indicates the specified heat capacity and density. Room temperature $400 \mathrm{~W} \mathrm{~m}-1 \mathrm{~K}-1$. [51]. The 
thermal conductivity of the compounds is then calculated as a function of density, heat dissipation and specific heat. The thermal conductivity equation (T) of the sample sheet is determined by lðТР $1 / 4$ аðТРСrðТЬrðТР, in which a (T), Crð T and $\rho$ (T) are heat dissipated, respectively. [52]. There is. Specify the specific heat capacity and density. The heat dissipation of sample paper based on the The well-known laser flash method Measured by LFA-447 (Netzsch, Germany). [53].

The sample sheet has a rectangular structure $200-300 \mathrm{~m}$ thick and ca long. P (T) is the heat dissipation respectively. Indicates specific heat capacity and density. $400 \mathrm{Wm}$ at room temperature $-1 \mathrm{~K}-1$. The thermal conductivity of the compounds is then calculated as a function of density, heat dissipation and specific heat. [54]. the thermal conductivity equation (T) of the sample sheet is determined by lðТЬ $1 / 4$ аðТЬСrðТЬrðТ, Where heat dissipation is a (Т), Сrð Т and $\rho$ (Т) respectively. Specify the specific heat capacity and density [55]. АðТЬСrðТЬrðТЬ where a (Т), Crð T and $\rho$ (Т) are the heat dissipaters respectively. Specify the specific heat capacity and density. [56]. The heat dissipation of the sample paper was measured by LFA-447 (Netzsch, Germany), which is based on the well-known laser flash method [57]. Sample sheet 200$300 \mathrm{~m}$ thick and ca long has a rectangular structure. $2 \mathrm{~cm}$. Specific heat capacity of Crð P T mixture depends on the weight ratio of the sample composition components. [58]. The thermal conductivity equation (T) of the sample sheet is determined by ІðТЬ $1 / 4$ аðТЬС̆ðТЬrðТЬ, where a (T), Сrð T and $\rho(\mathrm{T})$ are the heat dissipation, respectively [59]. Specify the specific heat capacity and density. The heat dissipation of the sample sheet was measured in the well-known laser flash mode by LFA-447 (Netzsch, Germany) [60].The sample sheet has a rectangular structure 200-300 m thick and ca long. $2 \mathrm{~cm}$. The specific heat capacity of the Crð P T mixture depends on the weight ratio of the sample composite components [61]. The thermal

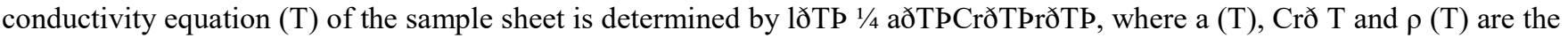
heat dissipation, respectively. Specify the specific heat capacity and density [62]. The heat dissipation of the sample paper was measured by LFA-447 (Netzsch, Germany), It is based on the well-known laser flash method. Sample paper 200-300 M thick and $2 \mathrm{~cm}$ long is rectangular in shape. Specific heat capacity of the Crð P T mixture depends on the weight ratio of the sample mixture components [63]

\section{Conclusion}

Thermal conductivity refers to the ability to conduct / transfer heat to a given object. It is usually denoted by ' $\mathrm{k}$ ', but Also referred to as ' $\lambda$ ' and ' $\kappa$ ' Contact this The level is called heat resistance, and Materials in high thermal conductivity heat sinks Used, at the same time materials with lower values are used as thermal insulators. Materials with low thermal conductivity are used in heat sinks, while materials with low values are used as thermal insulators. Graphene is an atom-thick carbon atom. Layer set in hexagonal lattice. This is the construction module of graphite (which, among other things, is used in pencil notes), but gropan only one notable object - with many astonishing properties, repeatedly receives the title of "miracle object". Nano composites are heterogeneous substances - so their properties determine the same Factors present in conventional compounds, i.e. properties, composition, structure and interface links. On the other hand, their setup is generally more complex than microcomputers, and this is especially true Polymer / layered silicate nanocomposites. A composite substance (also called a composite substance or abbreviated compound, It is a common name) an object made of two or more component materials. It's a A mixture of different ratios of carbon, oxygen and hydrogen to treat graphite with strong antioxidants Is obtained by. Acids that dissolve excess metals.

\section{References}

[1].Wejrzanowski, Tomasz, Mateusz Grybczuk, Marcin Chmielewski, Katarzyna Pietrzak, K. J. Kurzydlowski, and A. Strojny-Nedza. "Thermal conductivity of metal-graphene composites." Materials \& design 99 (2016): 163-173.

[2].Nair, Vineeth, Pratul Khosla, and M. Ramachandran. "Review on mechanical properties of various natural fibers reinforced composites." Research Journal of Pharmaceutical Biological and Chemical Sciences 7, no. 1 (2016): 20012004.

[3].Kalita, Kanak, Uvaraja Ragavendran, Manickam Ramachandran, and Akash Kumar Bhoi. "Weighted sum multi-objective optimization of skew composite laminates." Structural Engineering and Mechanics 69, no. 1 (2019): 21-31.

[4].Hou, Zhi-Ling, Wei-Li Song, Ping Wang, Mohammed J. Meziani, Chang Yi Kong, Ankoma Anderson, HalidanMaimaiti, Gregory E. LeCroy, Haijun Qian, and Ya-Ping Sun. "Flexible graphene-graphene composites of superior thermal and electrical transport properties." ACS applied materials \& interfaces 6, no. 17 (2014): 15026-15032.

[5].Manickam, Ramachandran. "Back propagation neural network for prediction of some shell moulding parameters." Periodica Polytechnica Mechanical Engineering 60, no. 4 (2016): 203-208.

[6].Ramachandran, M., Sahas Bansal, and Pramod Raichurkar. "Scrutiny of jute fiber poly-lactic acid (PLA) resin reinforced polymeric composite." Journal of the Textile Association 76, no. 6 (2016): 372-375.

[7]. Chu, Ke, Wen-sheng Li, and Hongfeng Dong. "Role of graphene waviness on the thermal conductivity of graphene composites." Applied Physics A 111, no. 1 (2013): 221-225.

[8].Pradeep, P., J. Edwin Raja Dhas, M. Ramachandran, and B. Stanly Jones Retnam. "Mechanical Characterization of jute fiber over glass and carbon fiber reinforced polymer composites." International Journal of Applied Engineering Research 10, no. 11 (2015): 10392-10396.

[9].Li, An, Cong Zhang, and Yang-Fei Zhang. "Thermal conductivity of graphene-polymer composites: Mechanisms, properties, and applications." Polymers 9, no. 9 (2017): 437.

[10]. Kurinjimalar Ramu; M. Ramachandran; M. Nathiya; M. Manjula " Green Supply Chain Management; with Dematel MCDM Analysis", Recent trends in Management and Commerce, 2(3),(2021): 8-15. 
[11]. Godbole, Nishant, Shajit Yadav, M. Ramachandran, and Sateesh Belemkar. "A review on surface treatment of stainless steel orthopedic implants." Int J Pharm Sci Rev Res 36, no. 1 (2016): 190-4.

[12]. Fegade, Vishal, Gajanan Jadhav, and M. Ramachandran. "Design, Modelling and Analysis of Tilted Human Powered Vehicle." In IOP Conference Series: Materials Science and Engineering, vol. 377, no. 1, p. 012215. IOP Publishing, 2018.

[13]. Koo, Minsoo, Jong-Seong Bae, SeongEun Shim, Dongmin Kim, Dae-Geun Nam, Jae-Won Lee, Gang-Woo Lee, Jeong Hyun Yeum, and Weontae Oh. "Thermo-dependent characteristics of polyimide-graphene composites." Colloid and Polymer Science 289, no. 13 (2011): 1503-1509.

[14]. Ramachandran, M. "Failure Analysis of Turbine Blade Using Computational Fluid Dynamics." International Journal of Applied Engineering Research 10, no. 11 (2015): 10230-10233.

[15]. Kalita, Kanak, Rakesh Chaudhari, and M. Ramachandran. "Mechanical characterization and finite element investigation on properties of PLA-jute composite." International Journal of Computer Applications 123, no. 13 (2015).

[16]. Shen, Xi, Zhenyu Wang, Ying Wu, Xu Liu, Yan-Bing He, and Jang-Kyo Kim. "Multilayer graphene enables higher efficiency in improving thermal conductivities of graphene/epoxy composites." Nano letters 16, no. 6 (2016): 3585-3593.

[17]. Yavari, F., M. A. Rafiee, J. Rafiee, Z-Z. Yu, and N. Koratkar. "Dramatic increase in fatigue life in hierarchical graphene composites." ACS applied materials \& interfaces 2, no. 10 (2010): 2738-2743.

[18]. Sharma, Malvika, and M. Ramachandran. "Development and characterization of fibre reinforced material based on potato starch and jute fibre." International Journal of Applied Engineering Research 10, no. 11 (2015): 10324-10327.

[19]. Tang, Zhenghai, Liqun Zhang, Wenjiang Feng, Baochun Guo, Fang Liu, and Demin Jia. "Rational design of graphene surface chemistry for high-performance rubber/graphene composites." Macromolecules 47, no. 24 (2014): 8663-8673.

[20]. Su, Liwei, Zhen Zhou, Xue Qin, Qiwei Tang, Dihua Wu, and Panwen Shen. "CoCO3 submicrocube/graphene composites with high lithium storage capability." Nano Energy 2, no. 2 (2013): 276-282.

[21]. Chinnasami Sivaji; M. Ramachandran; Kurinjimalar Ramu; Soniya Sriram "A Review on Weight Process Method and Its Classification", Data Analytics and Artificial Intelligence, 1(1), (2021):01-08

[22]. Luong, Duy Xuan, Kaichun Yang, Jongwon Yoon, Swatantra P. Singh, Tuo Wang, Christopher J. Arnusch, and James M. Tour. "Laser-induced graphene composites as multifunctional surfaces." ACS nano 13, no. 2 (2019): 2579-2586.

[23]. Gadde Mehar Chaitanya, M.P.Jenarthanan, C. Sathiyaraj, "A Review on Glass fibre Reinforced Composites with Different Matrix", REST Journal on Emerging trends in Modelling and Manufacturing, 7(1), (2021):18-24.

[24]. R. Kurinjimalar, S. Vimala, M. Silambarasan, S. Chinnasami. "A Review on Coir fibre Reinforced Composites with Different Matrix", REST Journal on Emerging trends in Modelling and Manufacturing, 7(2), (2021):25-32.

[25]. Mohan, Velram Balaji, Kin-tak Lau, David Hui, and Debes Bhattacharyya. "Graphene-based materials and their composites: A review on production, applications and product limitations." Composites Part B: Engineering 142 (2018): 200-220.

[26]. Kuilla, Tapas, Sambhu Bhadra, Dahu Yao, Nam Hoon Kim, Saswata Bose, and JoongHee Lee. "Recent advances in graphene based polymer composites." Progress in polymer science 35, no. 11 (2010): 1350-1375.

[27]. Li, J. L., Y. C. Xiong, X. D. Wang, S. J. Yan, C. Yang, W. W. He, J. Z. Chen, S. Q. Wang, X. Y. Zhang, and S. L. Dai. "Microstructure and tensile properties of bulk nanostructured aluminum/graphene composites prepared via cryomilling." Materials Science and Engineering: A 626 (2015): 400-405.

[28]. Amol Lokhande, C. Venkateswaran, M. Ramachandran, S. Chinnasami, T. Vennila."A Review on Various Implications on Re engineering in Manufacturing", REST Journal on Emerging trends in Modelling and Manufacturing, 7(3), 2021:70-75.

[29]. Huang, Ke-Jing, Lan Wang, Jing Li, and Yan-Ming Liu. "Electrochemical sensing based on layered MoS2-graphene composites." Sensors and Actuators B: Chemical 178 (2013): 671-677.

[30]. M. Amudha; M. Ramachandran; Chinnasami Sivaji; M. Gowri; R. Gayathri "Evaluation of COPRAS MCDM Method with Fuzzy Approach", Data Analytics and Artificial Intelligence, 1(1), (2021):15-23.

[31]. Huang, Ke-Jing, Lan Wang, Yu-Jie Liu, Yan-Ming Liu, Hai-Bo Wang, Tian Gan, and Ling-Ling Wang. "Layered MoS2-graphene composites for supercapacitor applications with enhanced capacitive performance." International journal of hydrogen energy 38, no. 32 (2013): 14027-14034.

[32]. Walker, Luke S., Victoria R. Marotto, Mohammad A. Rafiee, Nikhil Koratkar, and Erica L. Corral. "Toughening in graphene ceramic composites." ACS nano 5, no. 4 (2011): 3182-3190.

[33]. Amol Lokhande; C. Venkateswaran, M. Ramachandran, C. Sathiyaraj, K. Nathiya, "Recycling Process Impact in Current Scenario Manufacturing A Study", Recent trends in Management and Commerce, 2(1), (2021):20-25.

[34]. Vasileiou, Alexandros A., Marianna Kontopoulou, and Aristides Docoslis. "A noncovalent compatibilization approach to improve the filler dispersion and properties of polyethylene/graphene composites." ACS applied materials \& interfaces 6, no. 3 (2014): 1916-1925.

[35]. Gaikwad, Sakina Shajapurwala Tejashree, Mrs Vibhuti Khedekar, and Vishaka Karnad. "Creating Eco-consciousness about Natural Fabrics and Remodeled Garments amongst Youth."

[36]. Li, Weiping, Delong Li, Qiang Fu, and Chunxu Pan. "Conductive enhancement of copper/graphene composites based on high-quality graphene." RSC advances 5, no. 98 (2015): 80428-80433.

[37]. Tang, Long-Cheng, Yan-Jun Wan, Dong Yan, Yong-Bing Pei, Li Zhao, Yi-Bao Li, Lian-Bin Wu, Jian-Xiong Jiang, and Guo-Qiao Lai. "The effect of graphene dispersion on the mechanical properties of graphene/epoxy composites." Carbon 60 (2013): 16-27. 
[38]. P.K.Chidambaram, Amol Lokhande, M. Ramachandran, M. Nathiya, G. Mathivanan, " A study on Carbon Fiber Based Polymer Rein Force composites", REST Journal on Emerging trends in Modelling and Manufacturing, 7(3), (2021): 94-100.

[39]. S. Chinnasami, M. Ramachandran, P. Vidhya, M. Gowri " Study of Evaluation Based on Distance from Average Solution on Moyamoya Disease and Energy application" REST Journal on Emerging trends in Modelling and Manufacturing, 7(4), (2022):116-124.

[40]. Hong, Wenjing, Hua Bai, Yuxi Xu, Zhiyi Yao, Zhongze Gu, and Gaoquan Shi. "Preparation of gold nanoparticle/graphene composites with controlled weight contents and their application in biosensors." The Journal of Physical Chemistry C 114, no. 4 (2010): 1822-1826.

[41]. Sathiyaraj Chinnasamy, M. Ramachandran, Kurinjimalar Ramu, P. Anusuya "Study on Fuzzy ELECTRE Method with Various Methodologies" REST Journal on Emerging trends in Modelling and Manufacturing, 7(4), (2022):108-115.

[42]. Phiri, Josphat, Patrick Gane, and Thad C. Maloney. "General overview of graphene: Production, properties and application in polymer composites." Materials Science and Engineering: B 215 (2017): 9-28.

[43]. Sun, Yiqing, and Gaoquan Shi. "Graphene/polymer composites for energy applications." Journal of Polymer Science Part B: Polymer Physics 51, no. 4 (2013): 231-253.

[44]. Porwal, Harshit, Peter Tatarko, Salvatore Grasso, Jibran Khaliq, Ivo Dlouhý, and Mike J. Reece. "Graphene reinforced alumina nano-composites." Carbon 64 (2013): 359-369.

[45]. Khan, Zaheen U., Ayesha Kausar, Hidayat Ullah, Amin Badshah, and Wasid U. Khan. "A review of graphene oxide, graphene buckypaper, and polymer/graphene composites: Properties and fabrication techniques." Journal of plastic film \& sheeting 32, no. 4 (2016): 336-379.

[46]. Wang, Meng, XidongDuan, Yuxi Xu, and XiangfengDuan. "Functional three-dimensional graphene/polymer composites." ACS nano 10, no. 8 (2016): 7231-7247.

[47]. Araby, Sherif, Nasser Saber, Xing Ma, Nobuyuki Kawashima, Hailan Kang, Heng Shen, Liqun Zhang, Jian Xu, Peter Majewski, and Jun Ma. "Implication of multi-walled carbon nanotubes on polymer/graphene composites." Materials \& Design (1980-2015) 65 (2015): 690-699.

[48]. Ma, Jun, Qingshi Meng, Andrew Michelmore, Nobuyuki Kawashima, Zaman Izzuddin, Carl Bengtsson, and HsuChiang Kuan. "Covalently bonded interfaces for polymer/graphene composites." Journal of Materials Chemistry A 1 , no. 13 (2013): 4255-4264.

[49]. Khan, Zaheen U., Ayesha Kausar, Hidayat Ullah, Amin Badshah, and Wasid U. Khan. "A review of graphene oxide, graphene buckypaper, and polymer/graphene composites: Properties and fabrication techniques." Journal of plastic film \& sheeting 32, no. 4 (2016): 336-379.

[50]. Palermo, Vincenzo, Ian A. Kinloch, Simone Ligi, and Nicola M. Pugno. "Nanoscale mechanics of graphene and graphene oxide in composites: a scientific and technological perspective." Advanced Materials 28, no. 29 (2016): 62326238.

[51]. Bharathidasan, P., Dong-Won Kim, S. Devaraj, and S. R. Sivakkumar. "Supercapacitive characteristics of carbonbased graphene composites." Electrochimica Acta 204 (2016): 146-153.

[52]. M. Amudha; M. Ramachandran; Chinnasami Sivaji; R. Gayathri "A Study on Climate Change with Mayfly Algorithm Optimization", Recent trends in Management and Commerce, 2(3), (2021): 23-28.

[53]. Mohammed, A., Jay GnananandanSanjayan, W. H. Duan, and Ali Nazari. "Incorporating graphene oxide in cement composites: A study of transport properties." Construction and Building Materials 84 (2015): 341-347.

[54]. Bhanushali, R., and V. Karnad. "Dyeing of cotton and silk using vegetable kitchen waste: a step towards sustainability." S. No. Title of Paper Page No.: 28.

[55]. Dedhia, E. M., and V. S. Amembal. "A comparative study of magnesium chloride and sodium chloride as electrolytes for direct dyeing." Colourage 45, no. 8 (1998): 35-37.

[56]. Mulchandani, Neha, and Vishaka Karnad. "Application of zinc oxide nano particles using polymeric binders on cotton fabric." Research Journal of Textile and Apparel (2021).

[57]. Sompura, P., and V. Karnad. "POLYESTER-Product development from spun silk/polyester fabrics printed with geometric motifs and newsprints for home interiors." Colourage 58, no. 3 (2011): 40.

[58]. Jain, Ms Namrata, and Vishaka Karnad. "Online Forms for Data Collection and its Viability in Fashion and Consumer Buying Behavior Survey-A Case Study." (2017).

[59]. C. Venkateswaran; M. Ramachandran; Vimala saravanan; T. Vennila " A Study on Artificial intelligence with Machine learning and Deep Learning Techniques", Data Analytics and Artificial Intelligence, 1(1), (2021):32-37.

[60]. Mulchandania, Neha, and Vishaka Karnad. "Understanding users perspective for surgical apparel." Man-Made Textiles in India 46, no. 4 (2018).

[61]. Nisha Sherief; M. Anand; M. Ramachandran; Vidhya Prasanth, "A Review on Various Biofuels and its Applications", REST Journal on Emerging trends in Modelling and Manufacturing, 8(1), (2022):1-9.

[62]. Mulchandani, Neha, and Vishaka Karnad. "Application of Zinc Oxide nanoparticles on Cotton fabric for imparting Antimicrobial properties." International Journal for Environmental Rehabilitation and Conservation 11, no. 1 (2020): 110.

[63]. Nerkar, T., and V. Karnad. "ECO-FRIENDLY CLOTHING-Comparison of organic cotton, bamboo and linen towards product development for infant wear." Colourage 58, no. 7 (2011): 54. 